\title{
JOURNAL OF TELECOMMUNICATIONS AND INFORMATION TECHNOLOGY
}

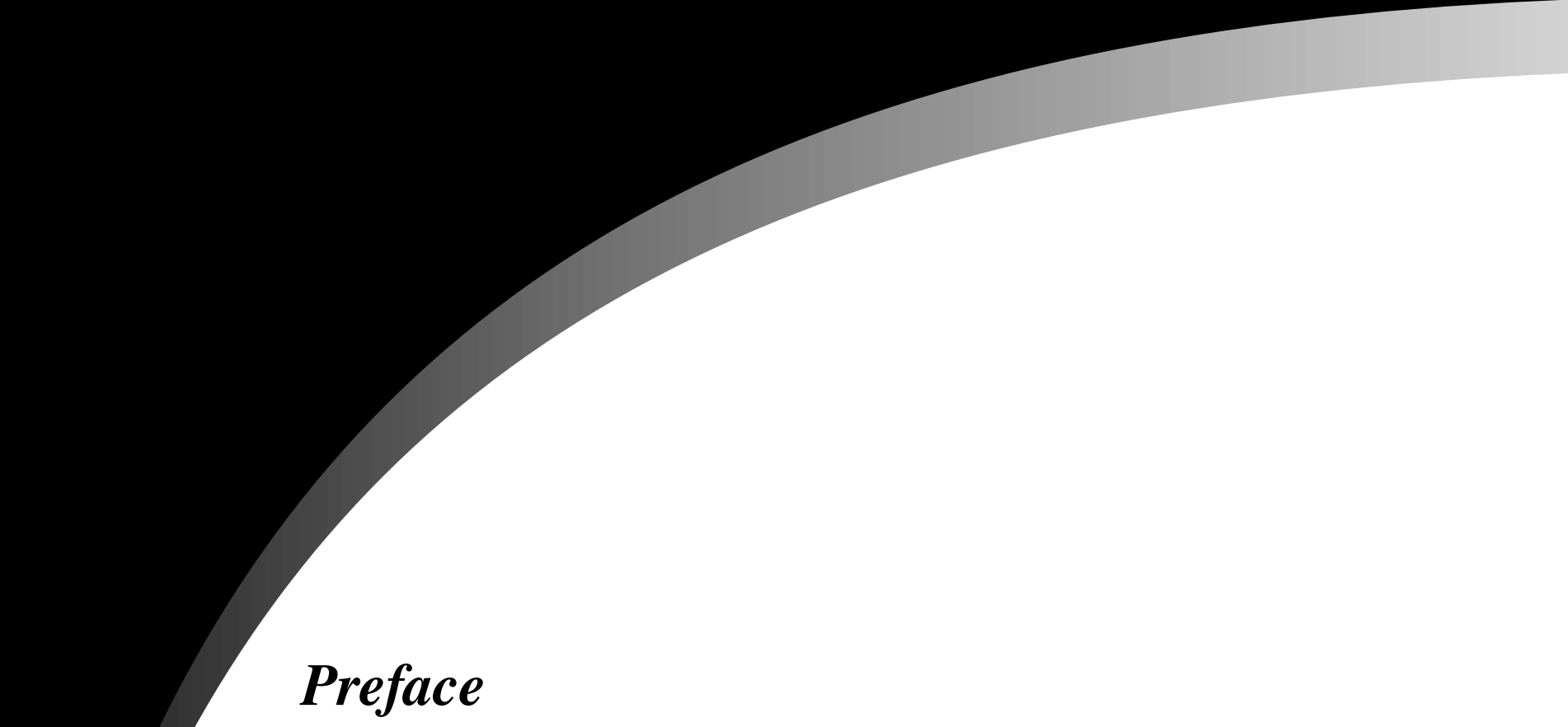

I am very delighted to present the next issue - third in 2019 - of the Journal of Telecommunications and Information Technology quarterly. This issue is the first to appear under my editorship - Adrian Kliks, affiliated to Poznan University of Technology. It is a great honor for me to serve as Editor in Chief for such a prominent international journal dealing with the recent achievements and trends in the broad ICT domain. At the same time, serving this role is a great and challenging commitment, as the minimum target is to at least maintain the high quality of all previous issues of JTIT. I would like to thank the previous Editors Prof. Paweł Szczepański, Krzysztof Borzycki and Marek Jaworski, all members of the Editorial Board, Handling Editors and Reviewers for their enormous work on shaping the high quality of this journal!

As the JTIT is an open access journal, we would like to gain more benefits from this great opportunity in the future. The review process will be kept unchanged, i.e., only the best papers that achieve a positive note in the rigorous, double-blind reviewing process can be accepted. Our goal as the Editorial Board is to minimize the review time, so the notification of the decision on each paper is delivered at the earliest possible time. I do hope that it will further enhance the reputation of the journal. If you consider being involved in the reviewing process, please do not hesitate to contact me!

The members of the Editorial Board, as well as the Reviewers, represent various countries from almost all continents, guaranteeing transparency and high quality of the entire review process. Moreover, as the journal is international in its scope and open-access in its nature, it covers up-to-date topics investigated in different places in the world, strengthening the opportunities for prospective collaboration even between distant research centers. I hope that due to this JTIT will be the space for exchange of results and ideas between scientists and experts both from academia and industry. 
JTIT is indexed in numerous databases, including such prominent ones as Scopus, Index Copernicus, BazTech, EBSCO, INSPEC or Google Scholar, just to mention a few of them. Of course, we are planning to further expand the visibility of our Journal.

Now, I think it is time to invite you to investigate deeply the ten, carefully selected papers collected in this issue. They cover selected research problems in the field of physical, medium access and network layers of communication systems, as well as deal with security issues. We hope you will enjoy this issue.

Adrian Kliks

Editor in Chief 